\title{
EFFECT OF VISUAL AND AUDITORY FEEDBACK EXERCISES ON SHOULDER FUNCTION IN ROTATOR CUFF TENDONITIS PATIENTS
}

\author{
Deimantė Jasnauskaitė-Gedrimė, Dovydas Gedrimas, \\ Aiva Karpavičienè, Albertas Skurvydas \\ Lithuanian Sports University, Kaunas, Lithuania
}

\begin{abstract}
Background. There is a lot of information in literature about rotator cuff strengthening, stretching, and postural correction exercises, which are recommended as a complex for tendonitis rehabilitation (Dong et al., 2015). There is lack of information about visual and auditory feedback exercises for shoulder functions in rotator cuff tendonitis.

Methods. The purpose of the study was to evaluate the effect of visual (VF) and auditory (AF) feedback exercises for shoulder functions in rotator cuff tendonitis patients. Thirty patients of Šiauliai rehabilitation centre were included in this study. Diagnostic tests, shoulder proprioception, posture, muscle strength (Lovett), functional muscle strength (Kendall), quality of life evaluations were performed before and after the study to evaluate the effect of $\mathrm{VF}$ and $\mathrm{AF}$ exercises.

Results. VF exercise group had a greater increase $(p<.05)$ in shoulder flexion abduction, internal and external rotation range of motion, proprioception, muscle strength results, also increased physical functioning, and decreased rolephysical and bodily pain in the quality of life evaluation. AF exercise group demonstrated a greater increase $(p<.05)$ in shoulder flexion abduction range of motion and muscle strength, bodily pain decreased in the quality of life evaluation.

Conclusions. After comparing the results we concluded that shoulder extension, abduction, internal and external rotation range of motion and proprioception, muscle strength and the quality of life increased $(p<.05)$ more in VF exercise group than in AF group. All in all, visual feedback exercises had greater effect onr shoulder functions and the quality of life compared to auditory feedback group. We would recommend including this type of exercises in rotator cuff tendonitis rehabilitation program.
\end{abstract}

Keywords: auditory feedback, visual feedback, quality of life, rotator cuff tendonitis.

\section{INTRODUCTION}

$\mathrm{R}$ otator cuff tendonitis is one of the most common pathologies diagnosed in shoulder region, which causes shoulder pain (Kolk, Yang, Tamminga, \& Van Der Hoeven, 2013). According to etiological studies, tears of rotator cuff occur for $25 \%$ of people older than 60 years old, and $20 \%$ of people older than 20 years old. Almost every person who has shoulder pain sooner or later seeks medical care (Hermans et al., 2013).

Active stabilizers of shoulder consist of rotator cuff muscles which surround humeral head (Dong et al., 2015). Tendonitis of these muscles occurs because of intrinsic and extrinsic factors.
Intrinsic factors are degenerative processes in tendons, which cause biomechanical, metabolic, and functional changes. Extrinsic factors are anatomically incorrect forms of acromion, kinematic changes between humerus and scapula bones, incorrect posture, incorrect force couples in shoulder and upper back, tight pectoral muscles, decreased mobility in posterior shoulder capsule (Mackenzie, Herrington, Horlsey, \& Cools, 2015).

Optimal proprioceptive information, which is essential for normal movement performance and correction, is about $150 \mathrm{~ms}$ (Salles et al., 2015). Visual feedback exercises described in literature 
about shoulder joint were only performed for posture correction where shoulder was affected indirectly (Weon et al., 2010). This type of treatment had good outcomes evaluating serratus anterior and upper trapezius muscle strength, also the authors described increased scapula - hummers rhythm.

Auditory feedback has the same effect as visual feedback, but in this case the therapist has to tell the patient what he/she needs to correct while performing a movement (Vogt, Pirrò, Kobenz, Höldrich, \& Eckel, 2010).

The problem of this study is: while there is a lot of information about rotator cuff strengthening, stretching, and posture correction exercises (Dong et al., 2015), there is lack of information about the effect of visual and auditory feedback exercises for rotator cuff tendonitis and shoulder functions.

The aim of a study was to evaluate the effect of visual and auditory feedback exercises for shoulder functions in rotator cuff tendonitis patients.

We hypothesised that visual feedback exercises would have a greater impact on shoulder functions than auditory feedback exercises because visual feedback makes it possible to correct errors faster.

\section{METHODS}

Participants. There were 30 subjects (17 men (mean age $41.47 \pm 15$ ) and 13 women (mean age $46 \pm 8.5)$ ) in this study, who were patients in Śiauliai Rehabilitation Centre. All participants had rotator cuff tendonitis diagnosed and treated conservatively. Inclusion criteria were: diagnosis confirmed by the doctor, pain lasting more than a month. Subjects were not included into this study if they had surgeries in the painful shoulder in the past or had any systemic diseases. Participants were randomly assigned into two groups: exercises with visual feedback (VF) (8 men, mean age $42.13 \pm$ 10.88 years, and 7 women, mean age $45.86 \pm 9.99$ years) and exercises with auditory feedback (AF) ( 9 men, mean age $40.89 \pm 18.6$ years and 6 women, mean age $46.17 \pm 7.33$ years). All subjects gave written consent for participation in the study and could get all information about changes in their condition during the study. Permission of Regional Ethics Committee was received on 201705 15, No. 17/12.

Examination. All subjects were examined two times: before and after the study. Examination began by performing special diagnostic rotator cuff tendonitis tests: painful arc, Neer's, HawkinsKennedy, Rent sign, Bear hug, empty/full can tests. Then posture examination was performed using Hoeger Posture Assessment Scale.

Range of motion and proprioception evaluation. Shoulder range of motion of flexion, extension, abduction, internal, external rotations were assessed using a goniometer (Hislop, Avers, \& Brown, 2013). Proprioception was examined immediately after the range of motion measurements, for all subjects blindfold was applied and the joint position sense testing began (Dover, Kaminski, Meister, Powers, \& Horodyski, 2003). Joint position sense testing first required the calculation of the target angle based on our range of motion measurements. We calculated the target angle by subtracting $10 \%$ of the total range of motion (external rotation + internal rotation or flexion + extension) from the specific range of motion being tested. We used a percentage of the total range of motion so that each subject would experience the same relative target angle. A sample calculation for external and internal rotation target angles is as follows: external rotation range of motion $=100^{\circ}$, internal rotation range of motion $=$ $80^{\circ}$; thus, the total range of motion $=$ external + internal rotation $=180^{\circ}$. To determine the target angle for external rotation, we take $10 \%$ of $180^{\circ}=18^{\circ}$; therefore, $100^{\circ}-18^{\circ}=82^{\circ}$ will be the target angle for external rotation for this subject. Likewise, for internal rotation, $80^{\circ}-18^{\circ}=62^{\circ}$; thus, $62^{\circ}$ would be the target angle for internal rotation for this subject. Target angles for all four movements were calculated. These target angles were then used for the subject to aim for during the repositioning required for the joint position sense testing. Specifically, we moved the subject's limb to the target angle and held it in place for 3 seconds. The subject was then told to relax and actively return the arm to the neutral starting position. During the internal/external rotation testing, the neutral position was achieved when the forearm was perpendicular to the table $\left(0^{\circ}\right.$ of shoulder rotation). During the flexion/extension testing, the neutral position was achieved when the subject's arm was relaxed at her side. Each subject was then instructed to actively return their arm to the target angle and to inform the investigator when they felt they had reproduced the original target angle. The arm was held motionless while the angle measurement was recorded. The repositioning was repeated three times for each of the four movements. 
Error scores from the three repositioning trials of each movement were calculated as the difference between the repositioning trial angle and the target angle. The absolute value of this difference was averaged over the three trials and used in the statistical analysis.

Muscle strength evaluation. Manual muscle testing was performed using Lovett scale (Hislop et al., 2013). Also, functional muscle strength testing was performed using Kendall, McCreary, Provance, Rodgers, and Romani's (2005) recommendations. Quality of life evaluation was performed using Short Form 36 (SF-36) questionnaire.

Experiment. The experiment took place in Šiauliai Rehabilitation Centre Physiotherapy Room. The study lasted from 20171109 till 2018 03 19. All subjects were randomly assigned to two groups: the first group $(n=15)$ performed exercises with visual feedback in front of the mirror, and the second group $(n=15)$ - using auditory feedback exercises, with the therapist commenting the performance of exercises and telling how to correct mistakes. Exercises were intended to strengthen rotator cuff muscles, to increase shoulder range of motion, better scapular control. All exercises were performed using elastic resistance, free weights, and additional measures, such as balls and sticks. Physiotherapy procedures lasted for 3 weeks, 5 times per week, 30 minutes per procedure. All participants received outpatient treatment, which consisted of physical therapy, physiotherapy and massage procedures which were assigned by a rehabilitologist according to each individual case.

Statistical analysis. Mathematical statistics were performed using SPSS 25 software. When all data were collected, we calculated if all values had normal distribution level. All data were distributed normally. Statistical significance was calculated using $T$ test, statistical significance was considered when $p<.05$. Also we calculated arithmetic averages, standard deviations, percentage expression of results.

\section{RESULTS}

After comparing the results we found that in $\mathrm{VF}$ group, decrease in special test results was found in painful arc $(n=5)$, Rent sign $(n=6)$, Bear hug $(n=6)$ tests (Table 1$)$.

In AF group, decrease in special test results was found in painful arc $(n=6)$ and Bear hug $(n=$ 7) tests. Results of posture in both groups showed no changes. Comparison of the range of motion results show (Figure 1) that in both $\mathrm{VF}$ and $\mathrm{AF}$ groups statistically significant $(p<.05)$ increase was found in shoulder flexion, abduction, external and internal rotations. Greater increase was found in VF group: flexion increased by $22.1 \%$ (from $104.53 \pm 44.88^{\circ}$ to $142.6 \pm 35.21^{\circ}$ ), abduction - by $22.58 \%$ (from $86.47 \pm 40.57^{\circ}$ to $120.6 \pm 38.24^{\circ}$ ), external rotation - by $40.39 \%$ (from $31.2 \pm 25.03^{\circ}$ to $52.47 \pm 21.4^{\circ}$ ), and internal rotation - by $45.57 \%$ (from $37.93 \pm 32.52^{\circ}$ to $64.4 \pm 21^{\circ}$ ) more than $\mathrm{AF}$ group.

Proprioception results show that shoulder flexion position sense statistically significantly $(p<$ .05 ) increased in VF group. Increase was greater by $28.91 \%$ (from $85.6 \pm 46.01^{\circ}$ to $120.27 \pm 35.22^{\circ}$ ) in VF group than in AF group (Figure 2). Position sense of shoulder extension also statistically significantly ( $p$ $<.05$ ) increased in VF group. Increase was greater by $31.6 \%$ (from $23.93 \pm 13^{\circ}$ to $35.73 \pm 13.48^{\circ}$ ) in $\mathrm{VF}$ group than in AF group. Position sense of shoulder external rotation statistically significantly $(p<.05)$ increased also in VF group. Increase was greater by $44.49 \%$ (from $25.4 \pm 20.55^{\circ}$ to $40.13 \pm 17.9^{\circ}$ ) in VF group than in AF group. Position sense of shoulder internal rotation statistically significantly
Table 1. Positive outcomes of tendonitis special tests
Note. Numbers show how many patients had positive diagnostic test outcome in that group. For example, 8 means that out of 15 patients, 8 had positive test outcome. We evaluated the decrease of these outcomes after the study. The greater the decrease, the better the effect of the therapy.

\begin{tabular}{|l|l|l|l|l|}
\hline \multirow{2}{*}{\multicolumn{1}{|c|}{ Test }} & \multicolumn{2}{c|}{ VF group } & \multicolumn{2}{c|}{ AF group } \\
\cline { 2 - 5 } & Before & After & Before & After \\
\hline Painful arc & $n=13$ & $n=8$ & $n=14$ & $n=8$ \\
\hline Hawkins-Kennedy & $n=14$ & $n=11$ & $n=13$ & $n=10$ \\
\hline Rent sign & $n=14$ & $n=8$ & $n=14$ & $n=13$ \\
\hline Bear hug & $n=15$ & $n=9$ & $n=15$ & $n=7$ \\
\hline Full can test & $n=14$ & $n=11$ & $n=14$ & $n=11$ \\
\hline Empty can test & $n=14$ & $n=11$ & $n=14$ & $n=11$ \\
\hline
\end{tabular}




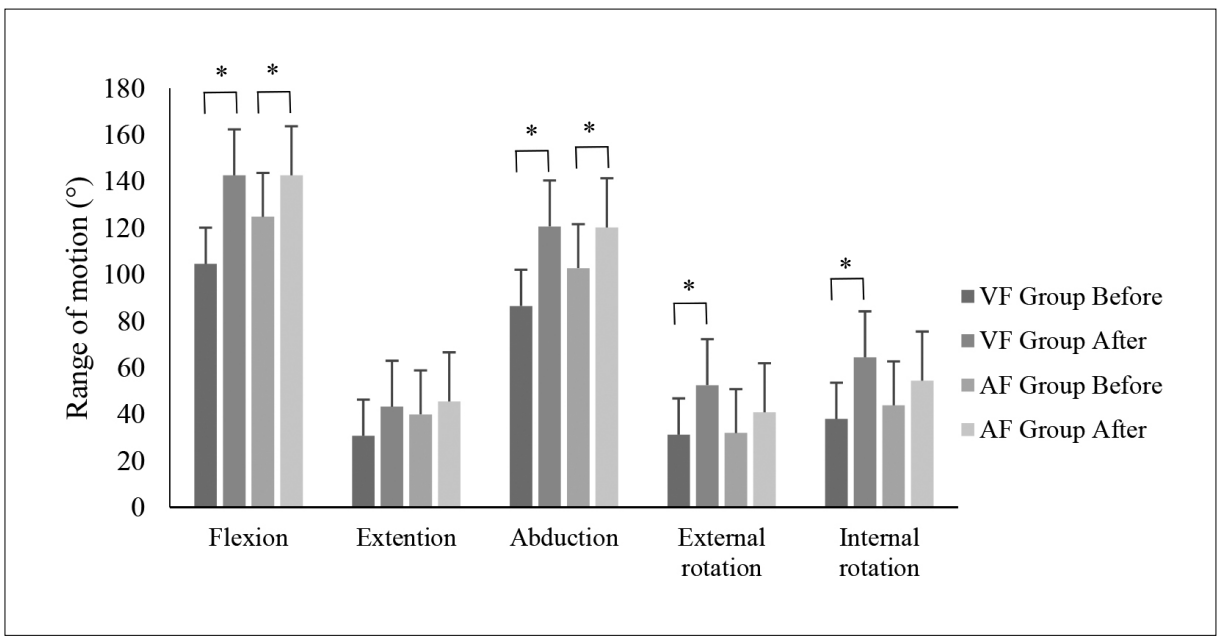

Figure 1. Difference in shoulder range of motion during the study

Note. All results in this figure are averages of every group subjects results, $* p<.05$, statistically significant difference between pre-experimental and postexperimental results.

$(p<.05)$ increased also in VF group. Increase was greater by $73 \%$ (from $33.27 \pm 31.22^{\circ}$ to $61.1 \pm 21.3^{\circ}$ ) in VF group than in AF group.

Results of manual muscle testing show (Figure 3) statistically significant $(p<.05)$ increase of muscle strength in shoulder flexion, abduction, as well as external and internal rotation in VF group. In AF, statistically significant $(p<.05)$ increase was found in shoulder flexion and abduction movements. Also, comparison of results between groups show that shoulder flexion strength increased by $21.96 \%$ (from $3.15 \pm 0.35$ to $4.2 \pm 0.56 ; p<.05$ ) more in
VF than AF group, shoulder abduction strength increased by $18.93 \%$ (from $3.08 \pm 0.26$ to $3.93 \pm$ $0.8 ; p<.05)$ more in VF group, shoulder external rotation strength increased by $45.9 \%$ (from $2.31 \pm$ 1.26 to $3.6 \pm 0.74 ; p<.05)$ more in VF group and internal rotation strength increased by $60.9 \%$ (from $2.23 \pm 1.25$ to $3.87 \pm 0.52 ; p<.05)$ more in $\mathrm{VF}$ group than in AF group.

Functional muscle strength testing results (Table 2) show that in VF group muscle strength increased statistically significantly $(p<.05)$ in middle trapezius by $17.8 \%$, deltoid $-15.6 \%$, seratus

Figure 2. Shoulder position sense in different movements

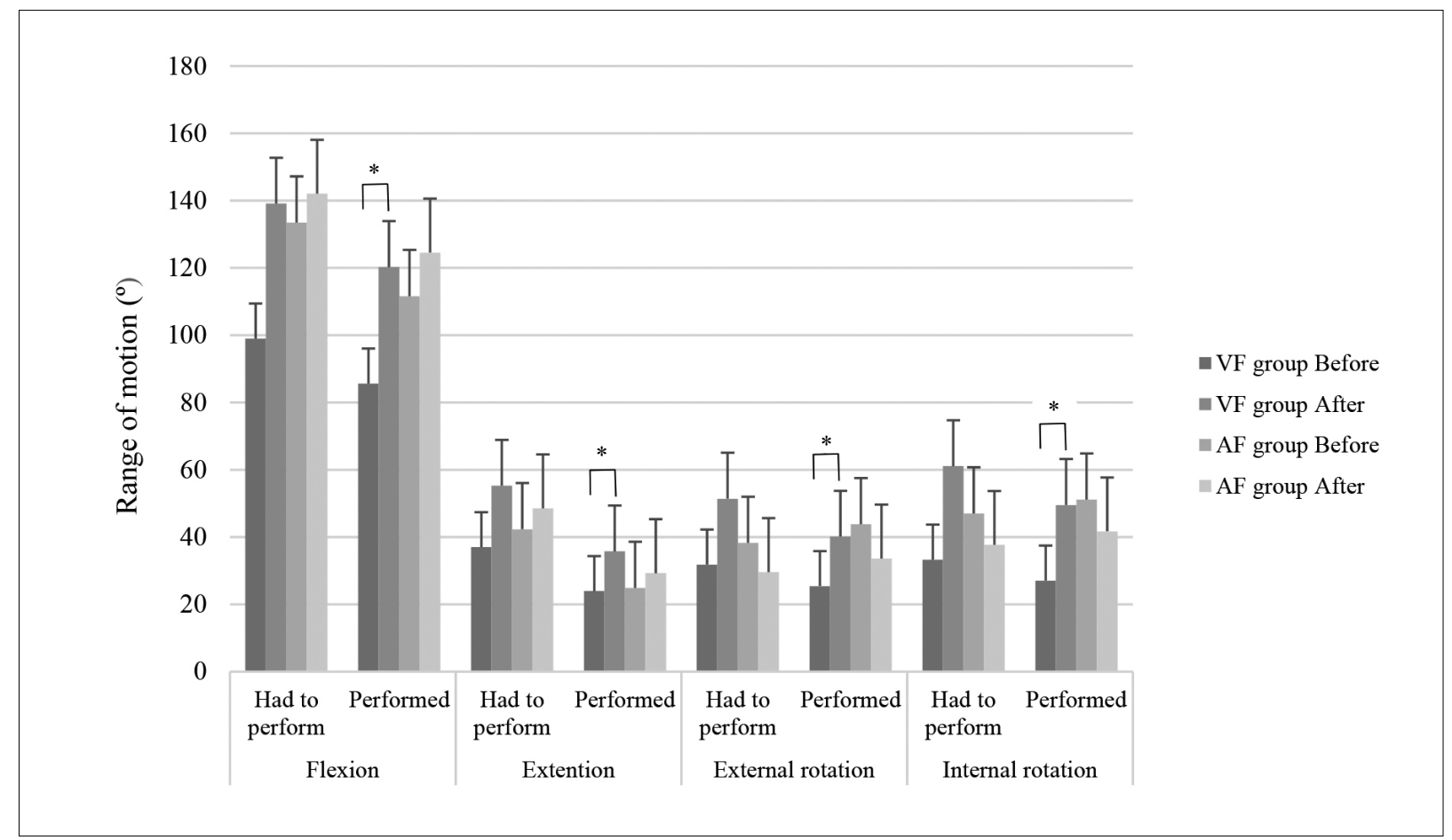

Note. The diagram shows the results of the required movement and the actual movement the subjects did. The lesser error - better proprioception, $* p<.05$, difference between pre-experimental and post-experimental results. 
Figure 3. Shoulder manual muscle strength testing results, measured in Lovett scale

Note. All values are averages of every group subjects. Results are measured on 1-5 Lovett scale. $* p<.05$, difference between pre-experimental and postexperimental results; $\# p<$ .05 , difference between VF and AF group results.

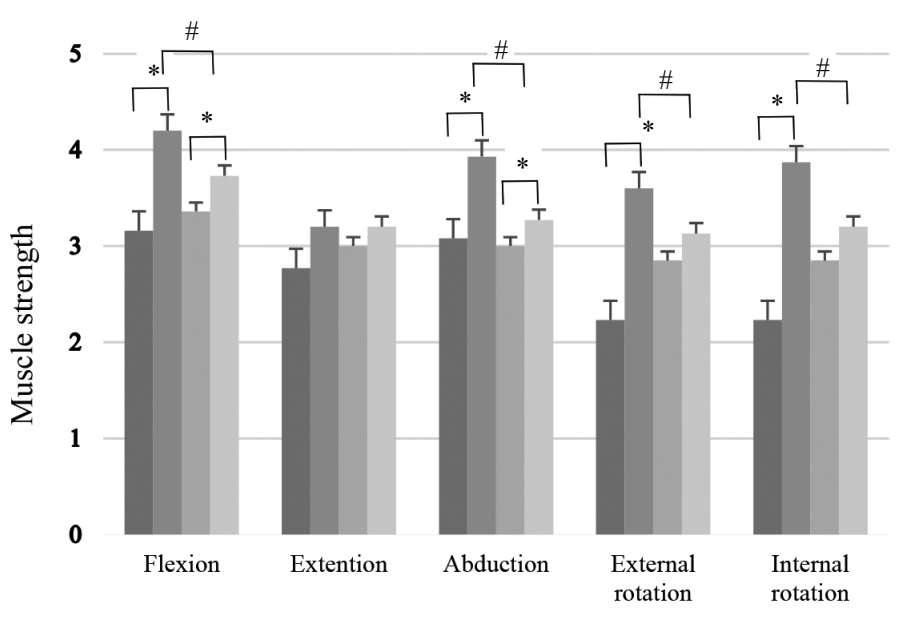

- VF Group Before

- VF Group After

$\square$ AF Group Before

IF Group After
Table 2. Results of functional muscle strength evaluation before and after the study

Note. $* p<.05$, statistically significant difference between pre-experimental and post-experimental results. All figures in table are averages of all group subject results.

Table 3. Quality of life evaluation in each SF-36 questionnaire subscale

Note. ${ }^{*} p<.05$, statistically significant difference between pre-experimental and post-experimental results. All figures in table are averages of all group subject results. $\# p<.05$, difference between VF and AF group results.

\begin{tabular}{|l|c|c|c|c|}
\hline \multirow{2}{*}{\multicolumn{1}{|c|}{ Functional muscle strength }} & \multicolumn{2}{c|}{ Before } & \multicolumn{2}{c|}{ After } \\
\cline { 2 - 5 } & VF group & AF group & VF group & AF group \\
\hline Upper trapezius muscle & $3 \pm 0.6$ & $2.8 \pm 0.4$ & $3.3 \pm 0.7$ & $3.1 \pm 0.6$ \\
\hline Middle trapezius muscle & $3 \pm 0.7$ & $2.8 \pm 0.5$ & $3.5 \pm 0.6^{*}$ & $3.2 \pm 0.4^{*}$ \\
\hline Lower trapezius muscle & $2,9 \pm 0.5$ & $2.8 \pm 0.4$ & $3 \pm 0.5$ & $2.9 \pm 0.4$ \\
\hline Deltoid muscle & $3 \pm 0.53$ & $2.8 \pm 0.4$ & $3.5 \pm 0.6^{*}$ & $3.1 \pm 0.3^{*}$ \\
\hline Pectoralis major muscle (clavicular part) & $2.6 \pm 0.5$ & $2.7 \pm 0.6$ & $2.7 \pm 0.5$ & $2.8 \pm 0.3$ \\
\hline Pectoralis major muscle (sternal part) & $2.7 \pm 0.4$ & $2.7 \pm 0.4$ & $2.8 \pm 0.4$ & $2.9 \pm 0.2$ \\
\hline Serratus anterior muscle & $2.7 \pm 0.6$ & $2.7 \pm 0.4$ & $3.1 \pm 0.4^{*}$ & 3 \\
\hline Rhomboid muscle & $2.5 \pm 0.5$ & $2.4 \pm 0.5$ & $2.7 \pm 0.4$ & $2.7 \pm 0.4$ \\
\hline Levator scapulae muscle & $2.6 \pm 0.5$ & $2.6 \pm 0.5$ & $2.8 \pm 0.4$ & $2.8 \pm 0.4$ \\
\hline Latisimus dorsi muscle & $2.9 \pm 0.5$ & $2.8 \pm 0.4$ & $3.4 \pm 0.6^{*}$ & $3.1 \pm 0.2^{*}$ \\
\hline
\end{tabular}

\begin{tabular}{|l|c|c|c|c|}
\hline \multirow{2}{*}{ SF-36 subscales } & \multicolumn{2}{c|}{ Before } & \multicolumn{2}{c|}{ After } \\
\cline { 2 - 5 } & VF group & AF group & VF group & AF group \\
\hline Physical functioning & $56.3 \pm 26.2$ & $67.3 \pm 24.9$ & $79.3 \pm 23.4 * ;$ & $68.3 \pm 22.5$ \\
\hline Role functioning/physical & $23.3 \pm 34.7$ & $16.6 \pm 22.5$ & $43.3 \pm 42.7 *$ & $20 \pm 27.1$ \\
\hline Bodily pain & $62.2 \pm 23.3$ & $63.7 \pm 18.5$ & $39.2 \pm 25.1 * ; \#$ & $48.1 \pm 14.9 *$ \\
\hline General health & $62.7 \pm 10.5$ & $51.7 \pm 8$ & $61.6 \pm 20.6$ & $55.7 \pm 13.1$ \\
\hline Vitality & $53 \pm 10.1$ & $43.6 \pm 9.5$ & $55.6 \pm 11.7$ & $46 \pm 9.5$ \\
\hline Social functioning & $45.9 \pm 19.2$ & $48.1 \pm 13.1$ & $54.1 \pm 11$ & $51.1 \pm 15.6$ \\
\hline Role functioning/emotional & $46.7 \pm 51.6$ & $31.1 \pm 36.7$ & $71.1 \pm 37.5$ & $57.8 \pm 42.7$ \\
\hline Mental health & $54.9 \pm 8.5$ & $53.9 \pm 8$ & $45.6 \pm 22.5 \#$ & $58.4 \pm 8$ \\
\hline
\end{tabular}

anterior $-15 \%$ and latisimus dorsi muscles $20.93 \%$. In AF group, statistically significant $(p<$ $.05)$ muscle strength increase was found in middle trapezius $-11.63 \%$, deltoid $-11.6 \%$, and latisimus dorsi muscles $-9.52 \%$.

Quality of life changes were found in both groups (Table 3). Bodily pain section of statistically significantly $(p<.05)$ decreased in both VF and AF groups. Also physical functioning increased, role functioning/physical decreased, and these changes also were statistically significant $(p<$ $.05)$ between groups. Also statistically significant $(p<.05)$ difference was found in the mental health section of the questionnaire. 


\section{DISCUSSION}

We found that exercises with visual feedback had a greater impact on shoulder functions: range of motion, proprioception, muscle strength, quality of life than exercises with auditory feedback in rotator cuff tendonitis patients. These exercises with visual feedback helped to increase more external and internal rotation range of motion and proprioception than in flexion and extension movements. The greatest difference was found in internal rotation movement, proprioception, and the external rotation movement.

Wilk, Hooks, and Macrina (2013) carried out a study about posterior shoulder capsule stretching to improve range of motion. Stretching was performed while scapula was stabilized, and stretching movement was internal and external rotation. Results after the study show that after stretching exercises, both rotation range of motion and mobility of posterior capsule statistically significantly increased.

Positive effect of visual feedback therapy is well known in treating phantomic pain using mirror, also in stroke patients (Thieme, Mehrholz, Pohl, Bahrens, \& Dohle, 2013). In our study pain decreased and shoulder function was better in VF exercise group. This happened because subjects could control themselves when they saw what they did in the mirror. This improved shoulder proprioception and helped to achieve better shoulder function. In their study, Foell, Bekrater, Bodmann, Diers, and Flor (2014) found that mirror therapy helped to reduce pain by $27 \%$ for participants. Our study results also show decrease of pain in the quality of life questionnaire in both auditory and visual feedback exercise groups.

Nodehi-Moghadam, Nasrin, Kharazmi, and Eskandari(2013) evaluated shoulder muscle strength, range of motion proprioception changes for athletes who performed throwing movements in their sport. Proprioception was measured with a goniometer when subjects had to perform movements and the difference was recorded. After the study, the results show that throwing movement performing athletes demonstrated statistically significantly greater external rotation movement. However, evaluating isometric muscle strength, they found that internal rotation force was greater than external rotation. Proprioception was better in the dominant hand. Our study results partly coincide because proprioception greater improved in VF group, but internal rotation movement proprioception was better than external rotation movement.
Boorman et al. (2014) evaluated the quality of life and prognosis after rotator cuff tear treated conservatively. All participants completed three month rehabilitation programme and after that were examined by an orthopaedic surgeon. Treatment was considered successful if both patient and surgeon agreed that surgery was no longer necessary because the quality of life and function increased. After that the study results showed that $75 \%$ of participants no longer needed surgery because the quality of life and shoulder function increased. Littlewood, Malliaris, Mawson, May, and Walters (2014) used SF-36 questionnaire to assess the quality of life. In this study participants were taught home exercises for rotator cuff tendonitis. After the study the authors concluded that all questionnaire subscale results increased, but better results were in the experimental group where exercises were performed at home. The greatest increase was found in role functioning/physical, bodily pain and role functioning/emotional subscales. Comparing with our results, we also found a great increase in role functioning/physical and bodily pain subscales. Also statistically significant difference was found in physical functioning subscale.

Roddy et al. (2014) described posture exercises to reduce pain for rotator cuff tendonitis patients. Our study found that posture after the study did not change, but scapulae stabilization and rotator cuff exercises had a positive effect on diagnostic tests outcome results: three test outcome results statistically significantly decreased in both groups. This could have happened because after exercises scapular control increased, rotator cuff muscle coordination and pressure in sub-acromial space decreased.

\section{CONCLUSIONS}

After comparing the results we concluded that shoulder extension, abduction, internal and external rotation range of motion and proprioception, muscle strength and the quality of life increased $(p<.05)$ more in VF exercise group than in AF group.

All in all, visual feedback exercises had a greater effect on shoulder functions and the quality of life compared to auditory feedback group. We would recommend including this type of exercises in rotator cuff tendonitis rehabilitation programme.

As there were no additional studies performed on this topic, we would recommend repeating this study with greater numbers of subjects and increasing the duration of the experiment to get more accurate results. 


\section{REFERENCES}

Boorman, R. S., More, K. D., Hollinshead, R. M., Wiley, J. P., Brett, K., Mohtadi, N. G., ... Bryant, D. (2014). The rotator cuff quality-of-life index predicts the outcome of non-operative treatment of patients with a chronic rotator cuff tear. JBJS, 96(22), 1883-1888. doi: 10.2106/JBJS.M.01457

Dong, W., Goost, H., Lin, X., Burger, K., Paul, C., Wang, Z., ... Kabir, K. (2015). Treatments of shoulder impingement syndrome: A prisma systematic review network meta-analysis. Medicine, 94(10). doi: 10.1097/ MD.0000000000000510

Dover, G. C., Kaminski, T. W., Meister, K., Powers, M. E., \& Horodyski, M. (2003). Assessment of shoulder proprioception in the female softball athlete. The American Journal of Sports Medicine, 31(3), 431437. https://doi.org/10.1177/03635465030310031801

Foell, J., Bekrater-Bodmann, R., Diers, M., \& Flor, H. (2014). Mirror therapy for phantom limb pain: Brain changes and the role of body representation. European Journal of Pain, 18(5), 729-739. doi: 10.1002/j.15322149.2013.00433.x

Hermans, J., Luime, J. J., Meuffels, D. E., Reijman, M., Simel, D. L., \& Bierma-Zeinstra, S. M. (2013). Does this patient with shoulder pain have rotator cuff disease? The Rational Clinical Examination systematic review. Jama, 310(8), 837-847. doi: 10.1001/jama.2013.276187

Hislop, H., Avers, D., \& Brown, M. (2013). Daniels and Worthingham's Muscle Testing-E-Book: Techniques of manual examination and performance testing. Elsevier Health Sciences.

Kendall, F. P., McCreary, E. K., Provance, P. G., Rodgers, M. M., \& Romani, W. A. (2005). Posture. In Muscles: Testing and function with posture and pain. 5th ed. (pp. 49-117). Baltimore: Lippincott Williams \& Wilkins.

Kolk, A., Yang, K. A., Tamminga, R., \& Van Der Hoeven, H. (2013). Radial extracorporeal shockwave therapy in patients with chronic rotator cuff tendinitis. Bone Joint J, 95(11), 1521-1526. https://doi. org/10.1302/0301-620X.95B11.31879.

Littlewood, C., Malliaras, P., Mawson, S., May, S., \& Walters, S. J. (2014). Self-managed loaded exercise versus usual physiotherapy treatment for rotator cuff tendinopathy: A pilot randomised controlled trial. Physiotherapy, 100(1), 54-60. https://doi.org/10.1016/j.physio.2013.06.001
Mackenzie, T. A., Herrington, L., Horlsey, I., \& Cools, A. (2015). An evidence-based review of current perceptions with regard to the subacromial space in shoulder impingement syndromes: Is it important and what influences it? Clinical Biomechanics, 30(7), 641648. https://doi.org/10.1016/j.clinbiomech.2015.06.001

Nodehi-Moghadam, A., Nasrin, N., Kharazmi, A., \& Eskandari, Z. (2013). A comparative study on shoulder rotational strength, range of motion and proprioception between the throwing athletes and non-athletic persons. Asian Journal of Sports Medicine, 4(1), 34. Retrieved from https:/www.ncbi.nlm.nih.gov/pmc/articles/PMC3685158/

Roddy, E., Zwierska, I., May, E., Jowett, S., Lewis, M., Stevenson, K., ... Foster, N. (2014). Sub-acromial impingement syndrome and pain: Protocol for a randomised controlled trial of exercise and corticosteroid injection (the support trial). Clinical Rheumatology and Osteoporosis, 25. doi: https://doi.org/10.1186/14712474-15-81

Salles, J. I., Velasques, B., Cossich, V., Nicoliche, E., Ribeiro, P., Amaral, M. V., \& Motta, G. (2015). Strength training and shoulder proprioception. Journal of Athletic Training, 50(3), 277-280. doi: https://doi. org/10.4085/1062-6050-49.3.84

Thieme, H., Mehrholz, J., Pohl, M., Behrens, J., \& Dohle, C. (2013). Mirror therapy for improving motor function after stroke. Stroke, 44(1), e1-e2. https://doi. org/10.1161/STROKEAHA.112.673087

Vogt, K., Pirrò, D., Kobenz, I., Höldrich, R., \& Eckel, G. (2010). PhysioSonic-evaluated movement sonification as auditory feedback in physiotherapy. In Auditory display (pp. 103-120). Springer Berlin Heidelberg. https://doi.org/10.1007/978-3-642-12439-6_6

Weon, J. H., Oh, J. S., Cynn, H. S., Kim, Y. W., Kwon, O. Y., \& Yi, C. H. (2010). Influence of forward head posture on scapular upward rotators during isometric shoulder flexion. Journal of Bodywork and Movement Therapies, 14(4), 367-374. doi: https://doi. org/10.1016/j.jbmt.2009.06.006

Wilk, K. E., Hooks, T. R., \& Macrina, L. C. (2013). The modified sleeper stretch and modified cross-body stretch to increase shoulder internal rotation range of motion in the overhead throwing athlete. Journal of Orthopaedic \& Sports Physical Therapy, 43(12), 891894. doi: 10.2519/jospt.2013.4990 